Aus dem Kgl. pathologischen Institut der Universität Kiel.

(Direktor: Geh. Rat Prof. Dr. Lubarsch.)

\title{
Beiträge zur Kenntnis der Chorionepitheliome beim Manne.
}

Von

Ewald Fritze,

3. Assistenten am Kgl. pathologischen Institut

der Lniversität Kiel, zurzeit im Felde.

Die Kenntnis ron dem Vorkommen chorionepithelialer Geschwülste beim Manne ist eine relativ junge. Der erste Fall, der unzweideutig als solcher erkannt und beschrieben wurde, ist der von Schlagenhaufer im Jahre 1902 veröffentlichte.

Seitdem sind noch eine Reihe weiterer Beobachtungen gemacht, durch die aber eine Lösung aller dieses Gebiet betreffenden Streitfragen noch nicht herbeigeführt ist. Schon aus diesem Grunde, mehr aber noch wegen der allgemein pathologischen Wichtigkeit der in Frage stehenden Gewächse und ihrer Beziehung zu den Missbildungen erscheint es nötig, weitere Erfahrungen zu sammeln, und jeden derartigen zur Beobachtung gelangenden Fall einer genauen, sachgemässen Untersuchung und Erörterung zu unterwerfen.

Aus diesem Grunde wurde ich von Herrn Geheimrat Lubarsch beauftragt, einen derartigen im pathologischen Institut zur Sektion gelangten Fall zu bearbeiten.

Bevor ich auf diesen Fall eingehe, erscheint es zweckmässig, auf Grund der bisher vorliegenden Erfahrungen eine Uebersicht über die ganze Frage des Chorionepithelioms beim Manne zu geben.

Das Chorionepitheliom beim Manne ist von Schlagenhaufer als eine spezifische Geschwulst erkannt und in Parallele gestellt mit dem Chorionepitheliom beim Weibe im Anschluss an Gravidität, eine Ansicht, die ron fast allen jetzigen Autoren anerkannt wird. Demgemäss lässt sich auch beim Manne derselbe Charakter dieser Geschwulst wie beim Weibe finden. Dem morphologischen Verhalten nach kann man mit Marchand bei diesem zwei Hauptformen, eine typische und atypische unterscheiden.

Bei der typischen findet man ,zusammenhängende epitheliale Massen in der bekannten Form der unregelmässigen vielkernigen Balken und verästelten Protoplasmamassen", die sich durch Vakuolenbildungen, einen 
Bürstenbesatz und zellige Einschlüsse auszeichnen können. Daneben findet man mehr oder weniger stark entwickelte polyedrische, helle Zellen vom Charakter der Langhansschen Zellschicht, die sich durch ihren zarten, durchscheinenden, membranös begrenzten, meist glykogenhaltigen Protoplasmaleib und einen regelmässig gestalteten, länglich-runden, bläschenförmigen Kern auszeichnen. Häufig kann man den „Charakter des Chorionepithels, wie er sich in der ersten Periode der Gravidität darstellt" wiederfinden in der gegenseitigen Anordnung der beiden Gewebselemente zueinander und dem gemeinsamen Verhalten zu der Umgebung. Wenn auch synzytiale Massen die Zellen vom Typus der Langhanszellen quer durchziehen können, so findet man das Synzytium doch speziell peripher gelagert, besonders dort, wo das Geschwulstgewebe an Blutungsherde anstösst. Das Verhalten gegen die Lmgebung kennzeichnet sich durch Gerinnungserscheinungen und Nekrose der Umgebung, durch Einwuchern der Geschwulstmassen in die Gefässe unter Zerstörung der Wandungen, das Auftreten grosser Blutungsherde, ausgedehnter Fibrinausscheidung und die Fähigkeit der Metastasenbildung auf dem Blutwege.

Bei der atypischen Form findet man isolierte Zellen vom Charakter der synzytialen Massen mit dem „Habitus der kompakten, stärker färbbaren und sehr unregelmässig geformten Zellen mit Kernen sehr verschiedener, oft ganz riesiger Grösse, intensiver, oft gleichmässiger Färbbarkeit". „Diese Elemente können auch vielkernige Klumpen bilden, aber es kommt in vielen Fällen nicht zur Entwicklung grösserer zusammenhängender Synzytiummassen. Uebergänge zwischen den beiden Hauptformen können dadurch entstehen, dass hie und da grössere Synzytien sich ausbilden."

Schliesslich muss noch als charakteristisch das Einwandern chorialer Zellen in die Umgebung nach Art der ektodermalen Wanderzellen hervorgehoben werden.

Die Frage des Chorionepithelioms beim Manne ist eine so eigenartige und auf den ersten Eindruck so fremdartige, dass es zur Sicherung der Diagnose der typischen, unzweifelhaften Form bedarf, um mit Sicherheit andere Tumoren auszuschliessen, bei denen ebenfalls chorionepithelähnliche Gebilde gefunden sind, die aber mit dem echten Chorionepitheliom nichts zu tun haben. Nun tritt aber das Chorionepithelion noch in atypischer Form auf. Hier ist die Abgrenzung der atypischen Form des Chorionepithelioms gegenüber ähnlichen Bildungen sehr schwer und nach Risel ist hier ,grosse Vorsicht geboten und es müssen alle sonstigen, für die Diagnose Chorionepitheliom bedeutsame Momente (Verhalten $\mathbf{z u}$ den Gefässen, Blutungen usw.) vorhanden sein, ehe man sich mit Wahrscheinlichkeit, aber auch nur mit mehr oder minder grosser Wahrscheinlichkeit, wird für die Diagnose aussprechen können“. Dementsprechend gehen auch 
bei einer Zahl der Fälle, auf die ich später zurückkommen werde, die Meinungen weit auseinander.

Die Zahl der Fälle, in denen man nun ein unzweifelhaft sicheres Chorionepitheliom gefunden hat, ist immerhin jetzt schon eine verhältnismässig nicht kleine, und ich beginne mit denen, die ihren primären Sitz im Hoden haben. Es ist dies die ganz überwiegende Mehrzahl der Fälle. und zwar findet man dort das Tumorgewebe allein oder in Terbindung mit einem Teratom,

Die Fälle, in denen der primäre Tumor lediglich chorioepitheliomatöse Bestandteile enthielt, sind die von Scott-Loncope, Frank (Fall I) und Oberndorfer-Seckel beschriebenen. Alle drei Fälle, besonders aber der letztere, sind später von Sigel genau referiert, es handelte sich in allen drei Fällen um ein typisches Chorionepitheliom mit Metastasenbildung. Hierzu kommt noch ein von Orton beschriebener sich gleichartig verhaltender Fall.

Weitaus die grösste Zahl der Beobachtungen zeigt neben dem ț̣ischen chorionepitheliomatösen Gewebe eine sichere Teratombildung. Es ist hier der klassische Fall ron Schlagenhaufer, ferner die ron Schmorl. Schmorl-Steinert, Risel (Fall I), Steinhaus-H. Askanazy-L. Pick, Salen-H. Askanazy-L. Pick, Hansemann-Holländer, Dillmann, Emanuel-Westenhöfer, Frank (Fall II), Kaufmann, Fischer und Risel zu nennen. Sämtlich hatten sie Metastasen gebildet und bei allen wurde in den Metastasen typisches Chorionepitheliom nachgewiesen, ausser bei dem letzten Fall von Risel, bei dem zwar Metastasen vorhanden, aber nicht untersucht werden konnten. Hierher rechne ich auch die Fälle von Wlassow. In fünf Fällen fand er im Hoden und in Metastasen chorionepitheliomgleiche Wucherungen, konnte Uebergänge in zylindrisehe, drüsenschlauchăhnliche Gebilde feststellen. Nur in einem Falle gelang es ihm, ausser Ento- und Mesoderm auch ektodermale Elemente festzustellen.

Wlassow ist der Meinung, es handle sich hier um ein chorionepithelähnliches Gebilde, das rom Entoderm abstamme. Risel hat diese Ansicht stark in Zweifel gezogen, indem er viele der zylinderförmigen Elemente in den genannten Tumoren als ektodermale Gebilde ansieht. Auch ich möchte mich dieser Ansicht anschliessen und annehmen, dass es sich hier um ein dreiblätteriges Teratom mit typischem Chorioepitheliom handelt. Weiter ist ron Glaserfeld ein Fall ron Chorionepitheliom beobachtet, in dem im primären Tumor im Hoden ron chorionepitheliomatösem Gewebe nichts nachzuweisen war, sich dagegen ein deutliches Teratom, epitheliale Zellwucherungen und Synzytium fanden, die Metastasen dagegen deutlich typisches Chorionepitheliom enthielten. Die epithelialen Zellwucherungen beschreibt Glaserfeld als alveolär gebautes Carcinom und lässt die synzytialen Massen aus Carcinomzellen entstanden sein. 
Eine neue Gruppe von Chorionepitheliomen bilden die, die ihren Sitz ausserhalb des Hodens haben. Als einwandfrei ist hier nur der Fall von Boström anzusehen, denn hier konnten in den'pathologisch-anatomisch genau untersuchten Hoden keinerlei Veränderungen nachgewiesen werden. Es fanden sich Herde von typischem Chorionepitheliom im Gehirn, in der Leber, Niere, Lunge und den retroperitonealen Lymphdrüsen. Wenn auch nicht mit Sicherheit nachgewiesen werden kann. welcher von diesen Herden der primäre war, so neigt Boström zu der Ansicht, dass in den retroperitonealen Lymphknoten der primäre Herd zu suchen sei. Zwei weitere Fälle, nämlich die von Askanazy und Hedinger, sind nicht mit Sicherheit hierher zu rechnen, da in beiden Fallen die Hoden nicht pathologischanatomisch untersucht werden konnten und nur feststeht, dass diese klinisch keine Veränderungen geboten haben. Askanazy fand ein typisches Chorionepitheliom ohne Teratombildung in der Zirbeldrüse, bei Hedinger fanden sich in den verschiedensten Organen zahlreiche Herde von typischem Chorionepitheliom, ohne dass einer von ihnen mit Sicherheit als primärer Tumor angesehen werden konnte. Ganz zweifelhaft steht in dieser Beziehung der Fall ron Bonney; bei dem die Hoden makroskopisch ohne Veränderung waren, sich ein Tumor im grossen Netz befand, der Metastasen in der Leber gebildet hatte und makroskopisch das Bild eines Chorionepithelioms bot. Risel konnte sich an mikroskopischen Präparaten nicht einmal daron überzeugen, dass es sich etwa nur um ein atypisches Chorionepitheliom handelt. Des weiteren beschreibt $R$ isel noch einen Fall, bei dem nur die Metastasen vorlagen, und von denen er angibt, dass er selber zweifelhaft sei, ob es sich hier auch nur um ein atypisches Chorionepitheliom gehandelt habe.

Ton einer Teratombildung war in allen ausserhalb des Hodens gelegenen Tumoren nichts nachzuweisen.

Mit den beiden letzten Fällen bin ich bereits zu der Gruppe derjenigen Geschwülste gekommen, die zwar ein chorionepitheliomähnliches Aussehen besitzen, über deren Zugehörigkeit zu dieser Gruppe aber noch sehr gestritten wird.

Ton älteren Fällen gehören hier die von Sarcome angioplastique her, wie sie ron Malassez-Monod, Carnot-Marie, Dopter, Kanthack und Eden beschrieben worden sind. Schlagenhaufer und Risel sind geneigt, dieselben zu den Chorionepitheliomen zu rechnen, während Sternberg und Mönckeberg sie für sarkomatöse Bildungen halten. Ein sicherer Aufschluss ist über die ersten drei Fälle nicht zu gewinnen, da die Beschreibungen $z u$ allgemein gehalten sind. Sternberg hat weiter einen Fall veröffentlicht, auf Grund dessen er die ganze Lehre des Chorionepithelioms beim Manne in Frage ziehen wollte, er deutete denselben als sarkomatöse Neubildung und nimmt an, dass die ganze Geschwulst von 
Endothelien ihren Ausgang nehme. Drei weitere Fälle veröffentlichte Mönckeberg, der diesen ebenfalls eine peri- oder endotheliale Entstehung zusprach.

Emanuel, Risel und Pick konnten sich über den Sternbergschen Fall dieser Meinung nicht anschliessen und Pick deutet ihn im Sinne seines später zu erwähnenden Epithelioma chorio-ectodermale. Bis auf den dritten Fall von Mönckeberg konnte in keinem dieser Geschwülste, die alle vom Hoden ihren Ausgang nahmen, etwas von Teratom festgestellt werden. Die Metastasen zeigten in allen Fällen den gleichen Befund wie der primäre Tumor, nur in dem dritten Fall von Mönckeberg war es nicht möglich, die Metastasen zu untersuchen. Ein weiterer von Chuvin beschriebener Fall ist uiberhaupt nicht einzureihen, da sich in dem betreffenden Referat nähere Angaben über den Tumor und seine Metastasen nicht vorfinden und im Referat nur gesagt ist, es handle sich um eine chorionepitheliomatöse Geschwulst.

Was die Genese des Chorionepithelioms beim Manne anbelangt, so vertritt Schlagenhaufer die Ansicht, dass es sich in Teratomen aus fötalen Eihäuten oder deren Rudimenten entwickle. Risel erweiterte später diese Ansicht Schlagenhaufers dahin, dass jedes fötale Ektoderm imstande sei, chorioepitheliomatöses Gewebe zu bilden, eine Meinung, die sich bis auf den heutigen Tag behauptet hat. Schwierig war nur die Beantwortung der Frage, wie es in den Tumoren, die keine teratomatösen Bestandteile erkennen lassen, zur Bildung chorionepitheliomatösen Gewebes kommen konnte. Hier hat sich am meisten die Ansicht ron Pick Geltung verschafft, dass es sich ursprünglich um ein Teratom gehandelt habe, dass aber die chorionepitheliomatösen Bestandteile die übrigen überwuchert und erstickt hätten. Risel hebt hervor, dass diese Deutung aber nur in denjenigen Fällen zulässig sei, in denen es sich um typisches Chorionepitheliom handle, dagegen, „wenn die hämorrhagischen Geschwulstmassen das Bild der atypischen Form bieten, wenn vielleicht Zellmassen vom Charakter der Langhansschen Zellen oder der vielgestaltigen plasmoidalen Gebilde von der ausgesprochen charakteristischen Form, wie sie sich an den Wucherungen der synzytialen Deckschicht des Chorionepithels finden, vollständig fehlen und das Bild durch Formen rom Aussehen der sogenannten Uebergangsformen beherrscht wird, zwischen denen hier und da einmal Gebilde vom synzytialen Charakter auftreten," müsse die teratomatöse Entstehung sehr in Frage gezogen werden. Glaserfeld, der sich bezüglich der Entstehung des Chorionepithelioms aus fötalem Ektoderm der Auffassung Risels anschliesst, nimmt für seinen, im primären Tumor kein Chorionepitheliom enthaltenden Fall eine Verschleppung junger, noch nicht differenzierter Ektodermzellen an und lässt aus diesen an Ort und Stelle der Metastase das chorionepitheliomatöse Gewebe entstehen. Ganz im 
Gegensatz zu diesen Auffassungen stellt sich, wie bereits erwähnt, Sternberg, der alle diese chorionepitheliomatösen Gebilde für nicht spezifisch hält und sie als Abkömmlinge einer endothelialen Wucherung bezeichnet. Er stützte sich dabei vor allen Dingen darauf, dass er unzweilelhaft einen Uebergang des Synzytiums aus dem Endothel nachweisen konnte, und auf die hauptsächlich perivaskuläre Lage der Tumorzellen. Wenn die Ansicht Sternbergs auch für manche der zweifelhaften Fälle in Betracht kommen kann, so ist sie doch sicher nicht zu verallgemeinern. Eine weitere Beobachtung, die auch gestattet, den Fall von Sternberg unter die chorionepitheliomatösen Geschwïlste einzureihen, machte Pick, indem er Uebergänge von Langhanszellen in polyedrische oder rundliche Zellen beschrieb, die teils in Komplexen, teils band-, drüsen- oder papillenförmig angeordnet waren. Pick sah solche Befunde in einem ron ihm beschriebenen Ovarialteratom, sowie in den Fällen von Steinhaus und Salen. Pick nahm an, dass es sich hier um eine Wucherung Langbansscher Zellen handle, die in hohem Masse fähig seien, ihren Zellcharakter zu ändern. Aehnliche Befunde wurden ron Emanuel, Dillmann und Frank (Fall II) in den oben angefiihrten Fällen beschrieben. Möglicherweise könnte man noch Fall II und III ron Bonney hier anführen, die jedoch nicht rollständig untersucht sind. Pick meint, dass das Vorhandensein von Synzytium, der Nachweis der teratoiden Natur des primären Tumors und der Zusammenhang der fraglichen Zellen mit ektodermalem Gewebe genüge, um die chorionepitheliale Natur dieser Zellmassen zu sichern.

Die Ansicht Sternbergs hat so die Lehre ron der ektodermalen Abstammung nicht erschüttern können, und wenn man mit Bonnet das Teratom aus einer befruchteten Polzelle oder einer abgeschnürten Blastomere herleitet, so sieht man, dass das Chorionepitheliom beim Hanne zum Träger in einem geschwisterlichen Verhältnis steht, im Unterschied zum Chorionepithel des Weibes, das aus dem befruchteten $\mathrm{Ei}$ der Trägerin herrorgeht und $z u$ ihr im Kindesverhältnis steht.

Ich komme nun zur Besprechung des von mir untersuchten Falles. Es handelt sich um einen 21 jährigen jungen Mann, bei dem etwa Mitte April 1913 der linke Hoden wegen Sarkoms im Krankenhause zu Aschaffenburg reseziert worden war. Die Geschwulst soll sich angeblich innerhalb eines Monats entwickelt haben.

Am 17. Mai 1913 wurde er in die Kgl. medizinische Kinik aufgenommen, wo er angegeben hat, dass er seit $4 \frac{1}{2}$ Wochen an Schmerzen im Leib und Stuhlverstopfung leide. Vorübergehend soll eine Besserung bestanden haben. Wenige Tage vorher war noch Würgen, das Gefühl des aufsteigenden Knäuels, schleimigblutiger Auswurf hinzugekommen. Ausserdem hatte sich noch Appetitlosigkeit eingestellt, er litt bäufig an saurem Aufstossen, war stark abgemagert, der Schlaf war schlecht, er bekam Beklemmungsgefühl, Schmerzen auf der Brust, Husten und Atemnot. 
In früheren Jahren war er nie krank, erbliche Krankheiten bestanden in der Familie nicht.

Status: Mittelgrosser Mensch in dürftigem Ernährungszustande.

Haut im Gesicht leicht gelblich, sonst ohne Oedeme und Exantheme. Schleimbäute gut durcbgeblutet, Skelett und Muskulatur obne Befund. Mund und Rachenorgane rot, Zunge feucht belegt, Gebiss vollständig. An der hinteren Rachenwand und im Munde zäher Schleim. Am Halse beiderseits Drüsen fühlbar. Brustkorb symmetrisch.

Lungen rechts vorn unten 6. Rippe, links vorn unten 4. Rippe, hinten beiderseits unterer Rand des 11. Brustwirbeldornfortsatzes. Spitzen beiderseits gleich hoch stebend, Grenzen gut verschieblich, lauter Lungenschall, reines vesikuläres Atmen ohne Nebengeräusche.

Herz: Grenzen oben unterer Rand der 3. Rippe, rechter Sternalrand, links etwas auswärts von der Mamillarlinie, Töne rein, Aktion regelmässig, nicht beschleunigt.

Abdomen: Druckempfindlichkeit des Epigastriums und etwas links vom Nabel, keine pathologische Resistenz, Leber reicht bis zum Rippenbogen, Milz nicht palpabel. Nieren 0. B. Albumen -, Saccharum --.

Augenbewegungen frei, Pupillen beiderseits gleich, prompte Licht- und Konvergenzreaktion, Kornealreflex herabgesetzt, Würgreflex vorhanden, Patellar- und Achillessebnenreflex lebhaft. - Bauch deckenreflexe lebbaft.

Patient macht einen äusserst nervösen, unruhigen Eindruck, er spricht stockend, ist etwas unklar im Intworten.

Im Sputum keine Tuberkelbazillen, auch bei Anwendung des Antiforminverfahrens. Probefrühstück: freie Salzsäure rorhanden, Gesamtazidität 36 .

Leukozyten 6700, Blutausstrich:

Polymorphkernige Leukozyten 72 pCt.,

Lymphozyten $25 \mathrm{pCt}$.,

Mastzellen $1 \mathrm{pCt}$.,

Uebergangsformen $1 \mathrm{pCt}$.,

Eosinophile Leukozyten 0 pCt.

Eine später nochmals wiederholte Sputumuntersuchung ergab keine Tuberkelbazillen. Der Blutgehalt dauerte an, man fand im Sputum Epithelien, Leukozyten und Streptokokken.

Die Röntgenuntersuchung ergab: Sehr starke Hilusdrüsenschwellung, infiltrative Prozesse in beiden Lungen.

Mit der Zeit verschlechterte sich das Befinden des Patienten immer mehr, die Schmerzen im Unterleib und in der Brust wurden stärker, es traten Symptome von seiten der Lunge in Form von feuchten Rasselgeräuschen auf, die Atmung wurde oberflächlicb, selbst bei grösster Anstrengung vermochte Patient nicht mebr tief zu atmen.

Da man eine Sarkomatose annahm, so wurde Patient mit Thorium und Röntgenstrahlen behandelt, der Zustand verschlechterte sich weiter, am Schädel traten weiche Knötchen auf, die sich vergrösserten, und am 22. Juni starb Patient unter dem Bilde eines Herztodes. 
Die am 23. Juni rorgenommene Obduktion ergab folgenden Befund (Obduzent Dr. Borchers):

Anatomische Diagnose: Operativer Defekt des linken Hodens. Zahlreiche Chorionepitheliommetastasen der linksseitigen inguinalen, der retroperitonealen, bronchialen und trachealen Lymphknoten, der Lunge, Leber, Niere, Nebenniere, Gehirn, Schädeldach, Därme und Magen.

Ausgedehnte fibrinöse Pleuritis, Bronchitis und Oedem der Lungen. Verfettung der Herzmuskulatur, Staungshyperämie der Milz und Nieren, Stauungs- und Fettleber.

Leiche eines stark abgemagerten jungen Mannes. Totenstarre vorbanden. Totenflecke ebenfalls. Der linke Hoden fehlt, im Skrotum eine lineäre, $4 \mathrm{~cm}$ lange Narbe.

Brusthöhle: Zwerchfellstand rechts 4. Interkostalraum, links 6. Rippe. Nach Eröffnung der Brusthöhle liegt der Herzbeutel in Kleinhandtellergrösse vor, die Lungen sind leicht zurückgesunken, nirgends verwachsen. In der Pleurahöble einige Kubikzentimeter einer leicht trüben, gelblichen Flüssigkeit. Die Pleura ist beiderseits träb, in den abhängigen Partien mehr als in den oberen mit gelben, abziehbaren Beschlägen bedeckt. Die Innenfläche des Herzbeutels ist glatt und spiegelnd, in ihm wenig klare, gelbe Flüssigkeit.

Das Herz ist von der Grösse der Faust der Leiche, von fester Konsistenz, das Epikard fettarm, zart und durchsichtig. Die Herzhöhlen enthalten Blut- und Speckgerinnsel, sind nicht erweitert, ibre Wandungen nicht verdickt. Die Farbe des Herzmuskels auf dem Durchschnitt ist bräunlichrot, das Endokard ist zart, der Klappenapparat intakt. Aorta weist Spuren von Sklerose auf. Die linke Lunge zeigt Vermehrung des Volumens, ihre Oberfläche ist knollig-höckerig, die Konsistenz unregelmässig, in den abhängigen Partien stärker als in den oberen. Die knolligen Vorwölbungen schwanken zwischen der Grösse einer Haselnuss und einer Walnuss. Die Farbe derselben ist teils gelblich, teils braunrötlich. Auf dem Durchschnitt sieht die Lunge rostbraun bis rot aus und ist durchsetzt von erbsenbis walnussgrossen blatreichen Knoten. Das dazwischen liegende Lungengewebe ist sehr feucht und lufthaltig, in den unteren Partien z. T. Iuftleer. Die Lungengefässe enthalten speckige Gerinnsel, die Bronchialschleimbaut ist dunkelrot mit eitrigem Schleim bedeckt. Auf der rechten Seite der gleiche Befund.

Die Hilus- und Trachealdrüsen sind stark vergrössert, sie stellen bis zu hühnereigrosse, weiche, dunkelrote Knollen mit glatter Oberfläche dar. Die Tonsillen sind nicht vergrössert, dagegen in leichtem Grade beide Lappen der Schilddrüse. Die Schleimhaut der Speiseröhre ist blass und glatt.

Bauchhöhle: Die Leber überragt den Rippenbogen um drei Querfinger, das Netz ist äusserst fettarm, überdeckt die Därme. Peritoneum glatt und spiegelnd.

Milz gehörig gross, von mässig fester Konsistenz, Kapsel glatt, Farbe auf der Schnittfläche blass, bräunlichrot.

Nebennieren zeigen mehrere erbsen- bis haselnussgrosse, weiche, dunkelrote Knoten, im übrigen sind Mark und Rinde deutlich zu unterscheiden. 
Nieren gehörig gross, von fester Konsistenz, Kapsel leicht abziehbar, Ober1läche glatt mit Ausnahme weniger erbsengrosser, dunkelroter, weicber Knötchen. Farbe auf der Schnittläche rötlich, Zeichnung scharf, Blutgehalt reichlich, Schleimhaut des Nierenbeckens blass und glatt.

Leber vergrössert, zeigt breite, flache, gelblich und bräunlich aussehende, rundliche Vorwölbungen, die sich auf der Schnittfläche scharf gegen das übrig gebliebene wenige Lebergewebe abheben und dunkelrot aussehen, das noch übrig gebliebene Lebergewebe sieht gelbbraun aus, Zeichnung undeutlich.

Die Gallenblase enthält dünnflüssige Galle, Gallenwege durchgängig.

Der Magen ist klein, seine Schleimhaut gefeldert, zeigt zwei linsengrosse, flache, ulzerierte Stellen mit erhabenen, geröteten und weichen Rändern. Die Schleimhaut des ganzen Darmes ist gerötet, leicht gequollen. Im Anfangsteil des Jejunum eine erbsengrosse Geschwulst ron dem gleichen blutigen Aussehen.

Die Retroperitonealdrüsen sind sehr stark vergrössert bis zur Hühnereigrösse, auf der Schnittfläche teils mehr dunkelrot, teils mehr grau gefärbt. Die Blasenschleimbaut ist blass und glatt.

Der rechte Hoden ohne Veränderungen.

Schädel und Gehirn: In der Kopfschwarte befinden sich mehrere bis erbsen. grosse, weiche, blutige Geschwulstknoten.

Knöchernes Schädeldach von normaler Dicke, symmetrisch gebaut.

Dura glatt, frei von Verwachsungen. Pia zart und durchsichtig.

Hirnwindungen gut gewölbt, Hirnsubstanz ohne Besonderes, ebenso Ventrikel und ihr Inhalt.

Im linken Schläfenlappen findet sich in der Hirnrinde eine erbsengrosse, dunkelrote Geschwulst. Mikroskopisch lässt sich noch Verfettung der Herzmuskulatur, Staungshyperämie der Milz und Nieren, sowie Stauungs- und Fettleber nachweisen.

Der resezierte Hodentumor wurde uns in liebenswürdiger Weise von Herrn Dr. Beyer-Aschaffenburg ïberlassen. Das Gewächs zeigt die Masse 10:6: $7 \mathrm{~cm}$. Die Aussenfläche wird von einer derben, fibrösen Kapsel gebildet, am oberen Pol, sowie in der Kapsel selbst sind Reste der Hodensubstanz in Form von dünnen Kanälchen sichtbar. Farbe und Konsistenz kann nicht mehr mit Sicherheit angegeben werden, da das Gewächs, in Formalin gehärtet, in meine Hände kam. Die Gefässe unter der Oberfliche sind stark gefiillt.

Die Schnittfäche bietet ein überaus buntes Bild. Vom Rande her durch die Tumormassen ziehende Bindegewebssepten geben derselben ein grobgefeldertes Aussehen. Die einzelnen durchschnittlich etwa haselnussgrossen Felder sind unter sich in ihrem Aussehen wieder recht verschieden.

In einigen findet man eine weisse, markige Substanz, stellenweise mit feinem, aber deutlichem guirlandenförmigen Relief. An anderen Stellen sieht man bräunliche Gebiete, die auf Blutungen hindeuten. Hier und da tindet man cystenartige Hohlräume, die leer oder mit einer granen, ge- 
romenen Masse gefïllt sind. Am oberen Pol fält ein besonders grosser. leerer, spaltförmiger Hohlraum auf.

Weiter finden sich Partien ron gelbbrauner Farbe, in denen das Gewebe ron krümeligem Aussehen ist und an nekrotisches Gewebe erinnert.

Bei der mikroskopischen Beschreibung des Tumors und seiner Metastasen sei rorweg bemerkt, dass, wem nichts Besonderes bemerkt ist, die Präparate mit Hïmatoxylin-Eosin gefürbt sind.

Ich beschränke mich darauf, die typischen Bilder zu schildern, zwischen denen es mannigfache Cebergänge gibt.

Die Kapsel des Hodentumors besteht aus kollagenem Bindegewebe, in dessen äusserer Lage man Reste ron Hodengewebe in Gestalt plattgedriickter, ein- oder mehrschichtiger Drüsenschläuche rom typischen Ausselen atrophischer Hodenkanälchen ohne Mitosen und mit verdickter Membrana propria findet. Der inneren Lage sind reichliche Mengen ron glatter Muskulatur beigemischt. Von der Kapsel aus ziehen Septen aus Bindegewebe und glatter Muskulatur in das Innere des Tumors.

Dort, wo man schon makroskopisch eine feine, guirlandenförmige Struktur sah, findet man mikroskopisch Bänder ron ein- oder mehrschichtigem Epithel, das meist zylindrische, vielfach auch kubische und polygonale Form hat und sich durch einen grossen, dunklen Kern, schwachgefärbten Protoplasmaleib auszeichnet, und zahlreiche Kernteilungsfiguren aufweist. Die Bänder liegen teils in geschlängelter, teils in papillärer Form. An den papillenartig angelegten Bündern kann man an deren Basis Zellen sehen, die platt sind, ein endothelartiges Aussehen besitzen, Hohlräume mit vereinzelten roten Blutkörperchen begrenzen und an einzelnen Stellen allmählich unter Vergrösserung ihres Kernes und Protoplasmaleibes in grosse, mehrkernige Zellen mit dunklem Protoplasma ïbergehen. Die Hohlräume sind teils spaltförmig, teils etwas weiter. Zwischen den Zellbändern und den endothelartigen Gebilden findet man kleinere Zellen mit blassem, länglichem oder rundem Protoplasmaleib von hellroter Farbe und kleinem Kern. An manchen Stellen füllen sie auch ganz die Maschen zwischen den epithelialen Zellbänderı aus. In diesen Zellansammlungen findet man wieder stellenweise eine Menge roter Blutkörperchen, die ron einem deutlichen, synzytialen Schlauch umgebeh sind und den Eindruck einer Kapillare mit synzytialer Wandung machen. An anderen Stellen findet man inmitten dieser letztgenannten Zellen, riesenzellartige, synzytiale Massen, in deren unmittelbarer Nähe man aber immer Blutgefässe oder zum mindesten eine Anzahl roter Blutkörperchen sieht. Die Zellen, in die die synzytialen Massen eingebettet sind, zeigen z. T. faserige Fortsätze, die sich nach van Gieson oder Mallory nicht färben lassen. Es hält daher schwer zu sagen, ob man sie für gewucherte Endothelien oder Bindegewebszellen halten soll. An rielen Stellen enthalten sie Ein- 
schlüsse von hyalinen Massen, die sich mit Eosin rot, mit Weigertscher Fibrinfärbung blau und mit Jod-Jodkali braun färben. Der Speichelverdauung unterliegen sie nicht. An wieder anderen Stellen liegen zwischen den Maschen Anhäufungen von feinkörnigen, hellroten, strukturlosen Massen, zwischen denen blau und dunkelrot gefärbte Klumpen liegen. Man hat hier den Eindruck von nekrotischem Gewebe.

An einer anderen, der eben beschriebenen makroskopisch ähnlichen Stelle finden sich die Zellbänder auch wieder in Papillenform, jedoch hier mit einem deutlichen, bindegewebigen Gerüst. Das Bindegewebe war teilweise von Rundzellen lymphozytären Charakters durchsetzt, z. T. ging es besonders am Ende der Zotten in ein zellreiches Gewebe iiber, dessen Zellen das Aussehen von Fibroblasten haben und wohl als solche aufzufassen sind. Auffällig ist hier wieder der Befund synzytialer Zellmassen, z. T. sind sie wieder an Blutgefässe gebunden, z. T. scheinen sie aber auch mit den Zellbändern in innigem Zusammenhang zu stehen. Aber man kann an solchen Stellen sehen, wie z. B. von einer nicht allzuweit gelegenen Blutkapillare, deren Epithel synzytial rerändert ist, an der Basis des Epithels, von diesem streng getrennt, ein synzytiales, dünnes Band zu den grösseren Haufen hinzieht und mit diesen in ebenso innigem Zusammenhange wie das Epithel steht. Weiter kann man aber auch an solchen Stellen mitten im Synzytium eine kreisrunde Lücke fiuden, die ganz von roten Blutkörperchen ausgekleidet ist und möglicherweise so eine Kapillarwand darstellt. Kernteilungsfiguren finden sich auch hier in reichem Masse.

An wieder einer anderen Stelle findet man fast das typische Bild eines Adenocarcinoms, in dem Schläuche, teils auch etwas weitere Hohlräume von zylinderförmigen Zellen gebildet und in einer fibrösen oder myxomatösen Grundsubstanz eingebettet liegen.

In einem anderen Gesichtsfelde sieht man mehr polygonale Epithelzellen, die teils in alveolärer Anordnung, teils in Strangform ebenfalls in einem fibrösen oder myxomatösen Gewebe liegen. Synzytiale Gebilde konnten an diesen, mit gefässlosem Stroma versehenen Partien nicht nachgewiesen werden.

Weiter wird an manchen Stellen die Grundsubstanz in der Hauptsache von eng aneinander liegenden, polygonalen, unregelnässig gestalteten, epithelartigen Zellen, mit unregelmässigen, blassen Kernen gebildet. Einzelne dieser Zellen enthalten hyaline Einschlüsse. In dieser Grundsubstanz sieht man nun Züge von zylinderförmigen Zellen eingestreut, die von derselben Beschaffenheit sind wie die oben beschriebenen und allmählich in die Grundsubstanz übergehen. Weiter erkennt man schon makroskopisch sichtbare zystische Hohlräume, die meist mit einer flockigen oder homogenen, leicht rötlich gefärbten Masse angefüllt sind. Begrenzt sind diese 
Hohlräume teils durch flache, glatte, teils durch kubische, teils durch zylindrische Zellen. Aber auch verschiedene Elemente machen manchmal die Wandung einer Cyste aus. So findet man nebeneinander deutliche, sezernierende Becherzellen, die einer dünnen Lage Bindegewebes aufsitzen, zylinderförmige Zellen und platte, epithelartige Zellen. In anderen Cysten wird die Wandung ausser von einem der genannten Zellelemente, auch nur ron Grundsubstanz gebildet, kurz, die verschiedenartigsten Elemente beteiligen sich an der Umrandung dieser Hohlräume.

Neben Cysten sind grosse Bluträume eingestreut, deren Wandung sowohl von endothelartigen, wie auch von Tumorzellen gebildet sind. Letztere haben am Rande dieser Herde hier und dort leicht synzytiale Veränderungen. Auch hier finden sich Riesenzellen mit dreizehn und mehr Kernen eingestreut, fast immer aber kann man Blut, und seien es auch nur wenige, scheinbar frei im Gewebe liegende Erythrozyten in der Lmgebung nachweisen. Úm einige Kapillaren mit erhaltenem Endothel sieht man einen ein- oder mehrschichtigen Kreis von zylinderförmigen Epithelzellen. An einigen Stellen hat man den Eindruck, es handle sich hier um grössere Gefässe, deren Gefässwandung von diesen Zellen durchsetzt seien. Das Einwuchern von Tumormassen in die Gefässe selbst unter Vordrängung und Zerstörung des Endothels kann man ebenfalls beobachten.

Dann tinden sich noch Zellen, deren Charakter unmöglich zu erkennen ist, ferner kleine Inseln kollagenen Bindegewebes. Weiter kann man besonders an Stellen mit, straffem, kollagenem Bindegewebe und glatter Muskulatur deutlich den teratomatösen Charakter der Geschwulst nachweisen, indem man Hohlräume sieht, die mit typischem, verhornendem Plattenepithel ausgekleidet sind. Als mesodermale Abkömmlinge findet man neben Bindegewebe und Muskulatur noch Knorpel, und als entodermale Abkömmlinde wieder Hohlräume, die z. T. mit typischem Zylinderepithel, z. T. mit Schleim sezernierenden Becherzellen ausgekleidet sind.

Das makroskopische Aussehen der Metastasen ist bereits beschrieben. In der Leber findet man in ihnen einen zentralen Blutungsherd, in dem man Fibrin, rote Blutkörperchen und nekrotische Massen unterscheiden kann. Nach dem Rande zu findet sich eine schmale Zone, in der Zotten und Stränge von hellen polyedrischen, scharfbegrenzten Zellen mit bläschenförmigen Kernen umgeben sind ron einem synzytialen dunkel gefärbten Protoplasmasaum mit Einschlüssen von roten Blutkörperchen, Vakuolen und einem Bürstenbesatz am freien Rande. Diese Gebilde tauchen in Bluträume ein, so dass man ein Bild hat, das dem der Chorionzotten in der Plazenta vollständig entspricht. Von diesen Randpartien erstrecken sich grosse Zellhaufen vom Typ der eben beschriebenen Langhanszellen und synzytiale Massen in den Blutungsherd hinein. Je tiefer erstere liegen, je mehr verändern sie ihr Aussehen und werden zu polygonalen unregel- 
mässigen Zellen mit stark rot gefärbtem homogenen Zelleib und einem verschieden grossen gleichmässig dunkelrot gefärbten Kern. Dazwischen liegen dann die ebenfalls rot gefärbten synzytialen Zellmassen mit blassen Kernen, Vakuolen und zelligen Einschlüssen. Dort, wo noch wenig veränderte Langhanszellen an Blutgerinnsel anstossen, kann man auch wieder hier und da synzytiale Umwandlung erkennen. Das umliegende Lebergewebe ist exzentrisch zusammengedriickt, in ihm findet man hier und da vereinzelte grosse synzytiale Zellen, die nach Art der ektodermalen Wanderzellen in den Kapillaren der Leberzellbalken liegen. Weiter kann man das Einwuchern der polygonalen Zellen in stärkere Gefässwände unter totaler Zerstörung derselben sehen. Wir haben hier also ein Verhalten gegenüber der Umgebung, das ganz dem des normalen Chorionepithels entspricht.

In sämtlichen anderen Netastasen finden wir die gleichen Verhältnisse wieder.

In der Niere liegen am Rande der Bhutungsherde ebenfalls schmale synzytiale Bänder und Langhanszellen, das gleiche Bild bietet die Nebenniere, in der noch besonders deutlich die grossen synzytialen Zellen in dem umgebenden Gewebe und am Rande der allmähliche Uebergang ron Langhanszellen in Synzytium zu sehen sind. Die Lunge bietet ein gleiches Bild, zeigt ausserdem in den umgebenden Alveolen ausgedehnte Blutungen. Auch hier kann man das Annagen der Gefässe deutlich erkennen. Einen eigentümlichen Befund bieten noch die retroperitonealen Lymphdriisen dar, hier findet man neben chorionepitheliomatösen Elementen ausgedehnte nekrotische Bezirke, an deren Rand man Zellen erkennen kann, deren Zellkern an Gestalt und Grösse dem der Langhanszellen gleicht, nur etwas dunkler gefärbt ist. Der Zelleib ist etwas dunkler gefärbt von äusserst zackiger Gestalt. Zwischen den einzelnen Stellen befinden sich klaffende Lücken, eine Interzellularsubstanz ist nicht rorhanden, man hat hier den Eindruck degenerativer Erscheinungen, doch lässt sich der Charakter dieser Zellen nicht sicher erkennen.

Ganz eigentümlich ist num das Vorkommen ron Zylinderepithel daselbst, das wie im Hoden, auch hier zı Bändern geordnet ist, allmählich in nekrotische Massen übergeht und ein dem im Hoden erhobenen Befunde ausserordentlich ähnliches Bild liefert. Auch makroskopisch kann man bereits eine Aehnlichkeit konstatieren.

Der negative Ausfall der Glykogenfürbung dürfte von keiner Bedeutung sein, da die Präparate alle in Formalin gehärtet waren und lange Zeit darin gelegen haben.

Dass es sich in diesem Falle zweifellos $u m$ ein echtes Chorionepithel handelt, geht aus den Metastasen unzweideutig herror. Alle die oben beschriebenen Kemzeichen für das 1ypische Chorionepitheliom finden wir ḷier 
in so rollkommener und ausgesprochener Weise, wie man es sich besser nicht wïnschen kann. Es fragt sich nur, wie der im Hoden erhobene Befund, nämlich das völlige Felılen chorionepitheliomatỏsen Gewebes, damit in Einklang zu bringen ist.

Der einzige Bestandteil der an Chorionepitheliom im Hodentumor erinnern könnte, ist das Synzytium und man muss daran denken, ob es sich hier etwa um eine atypische Form des Chorionepithelioms handelt. Das ist aber nicht anzunehmen, da erstens das Synzytium gegenüber den epithelialen Massen ganz in den Hintergrund tritt und nur in sehr geringem Masse vorhanden ist und seine Beziehungen zum Blutgefässendothel so innige sind, dass seine Abstammung von ihm nicht gut bezweifelt werden kann.

Auf Grund dieser Tatsache könnte man auf den Gedanken kommen, der Meinung Sternbergs beizutreten, das Chorionepitheliom rom Endothel abzuleiten. Dagegen muss mau aber die von Risel und Mönkeberg betonte Tatsache anführen, dass noch lange nicht jedes Synzytium chorionepithelialer Natur ist, und dass synzytiale Gebilde bei Sarkomen und Carcinomen, wie $z, B$. in dem Fall von Davidson, mehrfach beobachtet worden sind. Mehr Wahrscheinlichkeit würde die Ansicht Sternbergs ja gewinnen: wenn man eimen deutlichen Cebergang von Endothelien in typisches chorionepitheliomatöses Gewebe sähe, davon ist aber hier keine Rede. Und es unterscheidet sich das hier in Betracht kommende Synzytium nicht unwesentlich ron dem in den Metastasen durch blässeres Aussehen des Protoplasmas und durch die Kleinheit und Helligkeit eines grossen Teiles der Kerne. Wenn man nun noch den deutlich epithelialen Charakter der, dic Hauptmasse darstellenden, wuchernden Zellen ansieht, so liegt kein Grund ror, die hier wohl sicher zum grössten Teil vom Endothel stammenden Synzytiummassen als chorionepitheliomatös anzusehen und daraus weitere Schlüsse zu ziehen. Auch die hier und da beobachtete perivaskuläre Lagerung dieser Zellen zwingt nicht zur Annahme Sternbergs; da solche Bilder auch bei carcinomatös wuchernden Geschwïlsten vorkommen.

Sucht man nach anderen Elementen im Tumor, die einen Zusammenhang mit den Metastasen geben könnten, so fallen besonders die zylinderförmigen Epithelzellen auf. Man könnte daher versucht sein, mit Wlassow einen entodermalen Ursprung der ganzen Geschwulst anzunehmen. Und es gibt Bilder, die einen hierzu verleiten könnten, wenn man daran denki, dass Uebergänge von diesen zylinderförmigen Zellen im Synzytium vorhanden sind. Ich habe aber bei der Erwähnung dieser Stellen bereits hervorgehoben, wie zweideutig diese Bilder sind, und dass meistens gleichzeitig Beziehmoen zu Blutgelässen und Endothelien nachzuweisen sind. Wlassow fasst nun aber die symzytialen Massen als rasodestruktive Elemente auf und erklärt so ihr Verhalten zu den Gefïssen. Da man aber in diesem Falle zu deutlich den Uebergang aus ganz normalen endothel- 
artigen Zellen in Synzytium nachweisen kann, so dürfte diese Ansicht Wlassows sich hier nicht aufrecht erhalten lassen. Wenn sich nun auch an dieser oder jener Stelle sichere Uebergänge zwischen den zylinderförmigen Zellen und den synzytialen Massen einwandsfrei nachweisen liessen, was für einige Stellen mit mehr oder minder grosser Wahrscheinlichkeit zugegeben werden mag, so würde dies die Auffassung Wlassows auch noch nicht in Anwendung zu bringen gestatten, denn erstens können wuchernde Entodermzellen ebenfalls Synzytium bilden, was mit Chorioepitheliom nichts zu tun hat, und zweitens, was das Wichtigste ist, ist es gar nicht bewiesen, dass diese Zylinderzellen entodermaler Herkunft sind. Es kann sich hier sehr wohl um ektodermale Gebilde handeln, denn das Ektoderm tritt schon unter normalen Verhältnissen im Neuroepithel und Stratum germinativum in Zylinderform auf, und dann sind von Emanuel in seinem Falle deutliche Uebergänge von Langhanszellen in solche Bänder von Zylinderzellen beobachtet worden, wie wir sie hier vor uns sehen. Wenn es auch erst nach langem Suchen gelang, so haben wir doch in unserem Tumor sichere ektodermale Elemente nachgewiesen, und nichts steht der Auffassung im Wege, dass wir es auch bei den zylindrischen Zellbändern mit Wucherungen ektodermaler Natur zu tun haben. Es bieten diese Zellbänder auch nicht einmal das typische Bild von entodermalen Zylinderzellen, denn sie sind unter sich schon recht mannigfach gestaltet und liegen derartig mit kubischen und polyedrischen Zellen zusammen, dass mit Sicherheit anzunehmen ist, dass diese Zellsorten miteinander verwandt sind. Und es sind auch wieder zu diesen mehr kubisch gebauten Zellen Uebergänge von Langhanszellen in den Fällen von Salen, Steinhaus, Dillmann und von Hansemann beobachtet worden. Wir sind dadurch schon auf den richtigen Weg gewiesen, indem wir das gesamte Bild unseres Tumors im Sinne des Epithelioma chorioectodermale Picks erklären, der auf Grund der oben genannten Fälle seine Theorie aufgebaut und gestützt hat. Wir finden hier alle die von ihm aufgestellten typischen Bilder und Bedingungen: Das carcinomartige Wuchern von Epithelzellen in alveolärer, Cysten-, Bandund Schlauchform, das Vorhandensein eines Teratoms mit sicherem ektodermalem Gebilde und Synzytium. Letzteres dürfte allerdings nur zum geringsten Teil und auch dann noch fraglich in diesem Sinne verwandt werden, wie aus dem oben Gesagten hervorgeht.

Bemerkenswert ist ja allerdings, dass im Primärtumor trotz eingehendster Untersuchung überhaupt keine chorioepitheliomatösen Stellen gefumden wurden, während die meisten Metastasen ausschliesslich den Bau eines typischen Chorionepithelioms zeigten und nur die Metastasen der retroperitonealen Lymphknoten Stellen enthielten, die einigen Abschnitten des primären Gewächses glichen. Aber schliesslich ist das doch nichts sehr Ingewöhnliches, da wir ganz allgemein bei allen metastasierenden 
Gewächsen verschiedene Stufen der Zelldifferenzierung in primären und metastatischen Herden finden können und ja besonders bei den Teratoblastomen mannigfache Beispiele kennen, dass eine einseitige Entwicklung einer Zellart unter mehr oder weniger vollständiger Verdrängung anderer Zellarten erfolgt, wofür ja gerade die reinen Chorioepitheliome des Hodens und die Strumen des Eierstocks überzeugende Beispiele sind. Das Besondere unseres Falles dürfte nur darin bestehen, dass im Primärtumor noch gar keine Entwicklung von typischem Chorionepithel erfolgte, in den Metastasen dagegen meist vollkommen einseitige Differenzierung der verschleppten Tumorzellen zu Chorionepithelien stattfand.

Ich sehe nach dem oben Gesagten auch keine Veranlassung, meinen Fall im Sinne Glaserfelds zu deuten, d. h. die Wucherungen im Hoden als rein carcinomatöse anzusehen und das chorioepitheliomatöse Gewebe in den Metastasen aus jungen undifferenzierten Ektodermzellen entstehen zu lassen.

Auf Grund meines Falles scheint es mir auch zweifelhaft, ob nicht der Fall 3 von Mönckeberg in chorioepitheliomatösem Sinne zu deuten ist. Mönckeberg hatte keine Gelegenheit, Metastasen dieses Falles zu untersuchen. Die Beschreibung seines primären Tumors weist aber mit dem meinen derartige Aehnlichkeit auf, dass man auf den Gedanken kommen muss, es könne sich hier um einen analogen Fall handeln.

Wenn wir nach diesen Auseinandersetzungen zum Schluss noch unsere Kenntnisse über die Chorionepitheliome beim Manne zusammenfassen wollen, so können wir etwa folgendes feststellen:

1. Als Chorionepitheliome beim Manne dürfen mit Sicherheit nur diejenigen Fälle betrachtet werden, die in Bau und Verbreitungsart dem typischen Chorionepitheliom des Uterus vollkommen entsprechen.

2. Legt man diesen Satz der Beurteilung der bisher veröffentlichten Fälle zugrunde, so kann man mit dem ron mir beschriebenen Fall 30 Fälle von Chorionepitheliom beim Manne als sicher betrachten, einen (Fall 3 von Mönckeberg) als höchst wahrscheinlich, während man alle übrigen als äusserst zweifelhaft ansehen muss ${ }^{1}$ ).

3. Man kann weiter feststellen, dass das Chorionepitheliom beim llanne fast ausschliesslich seinen Ausgang nimmt vom Hoden; denn nur ein Fall (Bostroems) kann als sicherer ektoorchitischer betrachtet werden, während der von Askanazy wohl nur als selır wahrscheinlich anzusehen ist.

1) Als zweifelhaft sehe ich auch die von Bonney erwähnten Fälle von Spilsbury und Hicks und den von Lawrence an, während die beiden in der Londoner pathologischen Gesellschaft vorgezeigten Fälle von J. Bernstein wohl als sicher angesehen werden müssen. 
4. Unter diesen Chorionepitheliomen des Hodens kann man wieder folgende Gruppen unterscheiden:

a) reine Chorionepitheliome des Hodens mit reinen Chorionepitheliommetastasen (3 Fälle);

b) zwei- bis dreiblättrige teratoide Gewächse des Hodens mit Chorionepitheliommetastasen

a) mit Vorhandensein chorionepitheliomatöser Stellen im Iluttergewächs;

в) ohne sichere Befunde von Chorionepitheliom im Muttergewächs;

r) mit reinen Chorionepitheliommetastasen;

D) mit gemischten Metastasen.

5. Bezüglich der Entstehungsweise der chorionepitheliomatösen Bildungen erscheint es am wahrscheinlichsten, mit Risel anzunehmen, dass sie von fötalen Ektodermzellen her ihren Ursprung nehmen, denen allein die Fähigkeit zur Chorionepithelbildung zuzuschreiben ist.

Zum Schluss möchte ich Herrn Geheimrat Lubarsch für die bei der Anfertigung der Arbeit gegebene Anleitung und Unterstütung meinen verbindlichsten Dank geziemend aussprechen.

\section{Literaturverzeichnis.}

1. Askanazy, Hans, Zur Kenntuis der chorionepitheliomatösen Wucherungen in den Teratomen der Keimdrüsen. Dissert. Leipzig 1904. (Aus der Th. Landauschen Privatklinik in Berlin unter L. Pick.)

2. Askanazy, M. (Genf), Teratom und Chorionepitheliom der Zirbel. Verhandi. der Deutschen pathol. Gesellsch. 10. Tagung. Stuttgart 1906. S. 58.

3. Bonney, V., On chorious-epithelioma of congenital origin. Pathol. Soc. of London. May 1906. - Transactions of the Pathol. Society of London. 1907. Vol. 58. Part. 1. p. 9. (Nach Risel zitiert.)

4. Carnot, P. et Marie, R., Sarcome angioplastique. Bulletin de la société anatomique de Paris. Janvier 1898. p. 82.

5. Chuvin, Zur Kasuistik der Chorionepitheliome bei Männern. Med. Klinik. 1908. Nr. 31.

6. Debernardi, Beitrag zur Kenntnis der malignen Hodengeschwülste. Zieglers Beitr. Bd. 41.

7. Davidsohn, C., Chorionepitheliom und Magenkrebs, eine seltene Verschmelzung zweier bösartiger Geschwülste. Charité-Annalen. Jahrg. 29. S. 426.

8. Dillmann, H., Ueber einen Fall von Chorionepitheliom beim Manne. Zeitschr. f. Krebsforschung. 1905. Bd. 3. Heft 1. S. 61.

9. Dopter, M. Ch., Sur un cas de sarcome angioplastique. Archives de méd. expér. et d'anat. pathol. 1900. T. XII. p. 769 . 
10. Eden, T. W., Deciduoma malignum: A criticism. Transact. of the obstetr. society of London. 1897. Vol. XXXVIII. p. 162. (Auch Risel.)

11. Emanuel, R., Chorionepitheliom des Hodens. Vorläufige Mitteilung. Zeitschrift f. Geburtsh. u. Gynäkol, 1904. Bd. 51. H. 2.

12. Emanuel, R., Ueber chorionepitheliomatöse Wucherungen in Hodentumoren. Monatsschr. f. Geburtsh. u. Gynäkol. 1905. Bd. 21. H. 3. S. 602.

13. Fis cher, Teratom eines Bauchbodens mit chorionepitheliomatösen Wucherungen und Metastasen. Arbeiten a. d. pathol. Institut zu Tübingen. 1908. Bd.6.

14. Frank, R. T., Chorionepitheliomatous proliferations in teratomata, especially in those of testicle, with three new cases. The journ. of American med. assoc. 1906, Jan. 23 and Febr. 3. p. 248 und 343.

15. Glaserfeld, Ueber das Hodenteratom mit chorionepitheliomähnlichen Bildungen. Zeitschr. f. Krebsforschung. Bd. 9.

16. v. Hansemann, Demonstration eines Falles von Chorionepitheliom beim Manne. Verhandl. d. Gesellsch. f. Geburtsh. u. Gynäkol. zu Berlin. 12. Dez. 1903. Zeitschr. f. Geburtsh. u. Gynäkol. 1904. Bd. 51. H. 2. S. 400.

17. Holländer, Chorionepitheliom des Hodens (Fall 1 s. v. Hansemann). Chirurgenkongr. 1904. Bericht im Zentralbl. f. Chir. 1904. Beilage zu Nr. 27. S. 148 .

18. Kaufmann, Fall von typischem Chorionepitheliom in Hodenteratom und Metastasen. Lehrbuch. 6. Aufl. S. 927 und 928.

19. Kanthack, A. A. and Tigg, T. S., A case of carcinoma of the testis in a joung man, with metastatic deposits lying free in the heart and the inferior vena cava. 'liansact. of the Pathol. Society of London. 1896/97. Vol. XIVIII. p. 139 and Journ. of Pathol. and Bacteriol. 1898. Vol. V. p. 78.

20. Malassez, C. et Monod, Ch., Sur les tumeurs à myeloplaxes (sarcome angioplastique). Arch. de physiol. norm. et pathol. 1878. p. 375.

21. Marchand, F., Ueber das maligne Chorionepitheliom nebst Mitteilungen von zwei neuen Fällen. Zeitschr. f. Geburtsh. u. Gynäkol. 1898. Bd. 39. S. 173.

22. Mönckeberg, Ueber syncytiale Hodentumoren. Virch. Arch. 1909. Bd. 190.

23. Oberndorfer, Chorionepitheliom rom Hoden eines 34jährigen Mannes mit Metastasen in fast allen Organen. Gynäkol. Gesellsch. in München. 16. Mai 1907. Münchener med. Wochenschr. 1907. Nr. 26. S. 1307.

24. Orton, Report of a case of chorionepithelioma of the testicle. Journ. of med. research. 1908 . p. 219.

25. Pick, L., Zur Histogenese des Chorionepithelioms. Zentralbl. f. Gynäkol. 1903. Nr. 37. S. 1110.

26. Pick, L., Das Epithelioma chorioectodermale, ein Beitrag zu der Lehre der kongenital angelegten Geschwülste. Berliner klin. Wochenschr. 1904. Nr.7 u.8.

27. Pick, L., Zur Frage der Entstehung des Chorioepithelioms aus angeborener Anlage. Virchows Archiv. 1905. Bd. 180. Heft 1. S. 172-179.

28. Pick, L., Zur Lehre vom Epithelioma chorioectodermale. Zentrabl. f. Gynäliol. 1905. Nr. 18.

29. Pick, I., Noch einmal zur Entstehung des Chorionepithelioms und Epithelioma chorioectodermale aus liongenitaler Anlage. Zentralbl. f. Gynäkol. 1905. Nr. 20. S. 821. 
30. Risel, W., Ueber das maligne Chorionepitheliom und die analogen Wucherungen in Hodenteratomen. Arbeiten a. d. pathol. Institut zu Leipzig. 1903. Heft 1. II. Die chorionepitheliomartigen Wucherungen in Hodentumoren. S. $112 \mathrm{ff}$.

31. Risel, W., Zur Frage der chorionepitheliomähnlichen Geschwülste. (Zwei Fälle von Magencarcinom mit chorionepitheliomähnlichen Metastasen.) Zieglers Beitr. 1907. Bd. 42. S. 233.

32. Risel, W., Chorionepitheliome, chorionepitheliomartige Wucherungen in Teratomen und chorionepitheliomähnlicbe Geschwülste. Lubarsch-Ostertag Ergebn. 1907. Jahrg. 11. Abteil. 2. S. $928 \mathrm{ff}$.

33. Risel, W., Ein Hodenteratom mit chorionepitheliomatösen Bildungen. Verhandl. d. Deutschen pathol. Gesellsch. 1909.

34. Schlagenhaufer, Ueber das Vorkommen chorionepitheliom- und traubenmolenartiger Wucherungen in T'eratomen. Wiener klin. Wochenschr. 1902. Nr. 22 und 23.

35. Schlagenhaufer, Ueber das Vorkommen chorionepitheliom- und traubenmolenartigerWucherungen in Teratomen. Vortrag. Diskussionsbemerkungen von Albrecht, Boström, Marchand, Schmorl, v. Recklinghausen. Verhandl. d. Deutschen pathol. Gesellsch. 5. Tagung. Karlsbad 1902. S. 209.

36. Scott and Loncope, The report of a case of malignant tumor of the testicle, resimbling chorioepithelioma with metastases. Bulletin of the Amer. clin. laborat. of Pennsilvania Hospital. 1905, Jan. Nr. 2. p. 56.

37. Sigl, Ueber Chorionepitheliom des Hodens ohne Teratombildung. Aus der Prosektur des Krankenhauses rechts der Isar in München. 1910. S. 39.

38. Steinert, H., Ueber die embryonalen Geschwülste der Keimdrüsen und über das Vorkommen chorionepitheliomatöser Bildungen in diesen Tumoren. Virchows Archiv. 1903. Bd. 174.

39. Steinhaus, Ueber chorionepitheliomatöse Wucherungen beim Manne. Wiener med. Wochenschr. 1903. Nr. 17. S. 793.

40. Sternberg, Zur Kenntnis der chorionepitheliomartigen Wucherungen in malignen Hodenteratomen. Vortrag. Diskussionsbemerkungen von Westenhöfer, Hecker, v. Hansemann. Verhandl. d. Deutschen pathol. Gesellsch. 7. Tagung zu Berlin. 1904.

41. Sternberg, Ein peritkeliales Sarkom (Hämatangio-Endotheliom) des Hodens mit chorionepitheliomartigen Bildungen. Zeitschr. f. Heilk. Heft 14.

42. Wlassow, Ueber die Patho- und Histogenese des sogenannten Sarcoma angioplastique. Virchows Archiv. 1902. Bd. 109. Heft 2.

43. Wlassow, Zur Lehre von den embryonalen Geschwülsten, Patho- und Histogenese des Chorionepithelioma entodermale. Dissert. Moskau 1903. (Nach Risel zitiert).

44. Hedinger, Malignes typisches Chorionepitheliom bei einem 23jährigen Manne. Sitzungsbericht d. med. Gesellsch. Basel. 2. Sitzung vom 23. Januar 1913. 